\title{
Endoscopic Ultrasound-Guided Pancreatic Cyst Ablation
}

In the recent years, due to advancement and widespread availability of imaging modalities, the incidence of pancreatic cystic lesions (PCL) has increased considerably. ${ }^{[1]}$ This increased detection of asymptomatic but potentially malignant or malignant cystic lesion poses a difficult management dilemma. Pancreatic cysts can be classified into two groups as follows: (i) acute or chronic pancreatitis (acute fluid collections and pseudocysts without lining epithelium lining and no malignant potential) and (ii) cystic neoplasms lined by epithelium. The current diagnostic algorithm involves an accurate diagnosis of cystic epithelial lesions and thereafter accurate identification of malignant potential. The symptomatic cystic lesions or cysts with a malignant potential are offered surgical resection. However, surgical resection is associated with high morbidity $(20 \%-40 \%)$ as well as mortality $(\sim 2 \%) \cdot{ }^{[2-4]}$ Therefore, there have been concerted efforts to develop minimally invasive treatment modalities for treatment of these patients. Endoscopic ultrasound (EUS)-guided pancreatic cyst ablation (PCA) with ethanol and/or paclitaxel has been evaluated as a minimally invasive alternative to surgery in patients who are high-risk for or refuse surgery. ${ }^{[5,6]}$ The initial results have been encouraging with complete $(<5 \%$ of original cyst volume) or partial (5\%-25\% of original cyst volume) image-defined response documented in $60 \%-70 \%$ of patients and moreover, elimination of baseline cyst fluid DNA mutations has also been reported..$^{[6,7]}$ However, the effect of PCA on cyst sonographic morphology, cyst fluid cytology, and the quality and quantity of cyst fluid DNA is not available, and moreover, the long-term sustainability of the ablative effect of PCA is not known. In the news and views of this issue, we discuss two studies that have looked at these lacunae in the literature.

First, a prospective single center study from Indiana University Health Hospital evaluated the morphological as well as cytological changes along with a change in cyst fluid DNA after PCA on PCL that measured $10-50 \mathrm{~mm}$ in diameter and contained 5 or fewer septations. ${ }^{[8]}$ Majority of patients included in this needed surgical resection but surgery was refused either by patients or patient was declared unfit for surgery. Before, PCA EUS morphology (i.e. septations, cyst wall thickness, the presence of nodules) and maximal 2-dimension cross-sectional diameter was recorded. Thereafter, EUS-guided fluid aspiration was done, and quantity, viscosity, and color of fluid were recorded. The aspirated sample was also sent for cytology in all patients and carcinoembryonic antigen and molecular analysis (RedPath Integrated Technologies) in selected patients.
The protocol for PCA was variable depending on the year of enrolment as the patients were enrolled in different studies during various time periods. From 2004-2009, they underwent initial ablation with saline solution or ethanol alone (as part of a randomized trial) and from 2009 to 2014 they underwent PCA with ethanol plus paclitaxel (in a prospective cohort study). Thereafter, all patients underwent follow-up EUS 2-3 months later and during the years 2004-2009, diagnostic EUS was followed by fine-needle aspiration (FNA) for cytology and finally an index or second ethanol lavage (depending on initial randomization). Patients enrolled from 2009 to 2014 underwent follow-up diagnostic EUS, repeat EUS-FNA for cytology in all patients and molecular analysis (when possible) and finally repeat cyst ablation in patients with an initial suboptimal response. In all patients (regardless of initial ablation regimen), repeat computed tomography (CT), MRI, or EUS was performed 3-6 months later and then yearly.

The cyst volume was evaluated by two-dimensional (linear EUS) or three-dimensional (CT or MRI) measurements. Two-dimensional cyst volume was measured using formula $4 / 3 \pi \mathrm{r}^{3}$, where $r$ represents the radius of the maximal cyst by linear EUS image. Three-dimensional volume was calculated by the simplified formula $\mathrm{d} 1 \times \mathrm{d} 2 \times \mathrm{d} 3 / 2$, where $\mathrm{d} 1$, $\mathrm{d} 2$, and $\mathrm{d} 3$ represent the maximal diameters in the axial, coronal, and sagittal planes, respectively. The response was labeled as complete response (CR), partial response (PR), or persistent with $<5 \%, 5 \%$ to $25 \%$, and $>25 \%$ change of the original cyst volume, respectively. Molecular analysis was performed by clinically blinded laboratory personnel. DNA was isolated by using $200 \mu \mathrm{l}$ of pancreatic cyst fluid (Qiagen, Valencia, Calif, USA) and quantity measured by using spectrophotometer (NanoDrop, Willmington, Del, USA). DNA amplification was done by using polymerase chain reaction (PCR; i Cycler; BioRad, Hercules, Calif, USA).

The authors studied 36 patients (24 females) with a mean age of $69.1 \pm 12.2$ years. In $22(61.1 \%)$ patients, the cysts were located in the body and tail with the cyst size ranging from 10 to $50 \mathrm{~mm}$. The presumed clinical diagnosis was $16(44.4 \%)$ mucinous cystic neoplasms (MCN), 14 (38.9\%) branched intraductal papillary mucinous neoplasms (IPMN), 5 (13.9\%) SCA and 1 pseudocyst. EUS guided PCA was initially performed by using ethanol only in eight patients and in 28 patients by using a combination of ethanol and paclitaxel injection. Second PCA was performed in 17 patients, and third PCA was performed in only one 
patient. Repeat PCA was not performed in 18 patients due to decrease cyst size in 11 , acute pancreatitis in 3 , decreased cyst size with increased internal debris $(n=1)$, pseudocyst formation at gastric wall $(n=1)$, markedly increased internal debris $(n=1)$, and refusal $(n=1)$ after the first ablation. CR, PR and nonresponse were achieved in 19 out of 34 (56\%), 7 (21\%), and $8(23 \%)$ patients, respectively. A total of 54 PCA (First PCA in 36 patients + Second PCA in 17+ third PCA 1 patients) were performed, and 9 patients had procedure-related complication including pain abdomen in 4 patients, pancreatitis in 4 patients and hemorrhage in cyst in one patient.

Post-PCA, EUS was performed in 34 patients, and it showed an increase of cyst wall diameter in 23 of 34 patients, decrease in a number of cysts in 3 patients, loss of septations in 5 patients, increase in septations in 2 patients and loss of mural nodule in 5 patients. Intra-cystic debris developed in 8 patients and 1 patient developed a new mural calcification, whereas one patient developed both mural nodule loss and development of calcification. Post-PCA cytology assessed in 34 patients showed increased in epithelial cellularity in 9 patients, decrease or loss of an atypical cell in five patients and increase or newly developed atypical cell in 3 patients. Microscopically, post-PCA increase in cyst debris was observed in 12 patients, and fluid viscosity alteration was observed in 14 patients. No difference in sonographic or cytological changes was present between complete responders compared to those with a partial or nonresponse.

Preablation DNA quantity was evaluated in 20 patients who underwent PCA by using ethanol and paclitaxel, and it revealed mean DNA quantity and quality of $60.4 \pm 239.6 \mathrm{ng} / \mathrm{uL}$ (range 1.6-1,078.0) and $29.3 \pm 2.8$ (range 24.3-36.8) (Ct value), respectively. Post-PCA DNA quantity and quality was evaluated in 17 of these 20 patients and mean postablation DNA quantity and quality were $35.8 \pm 60.6 \mathrm{ng} / \mathrm{uL}$ (range 1.5-255.4) and $27.1 \pm 2.9$ (range 23.8-32.9), respectively. When classified by imaging response, postablation DNA amount increased in 12 out of $17(70.6 \%)$, including 10 of $12(83.3 \%)$ in the CR group, whereas overall, postablation DNA Ct value decreased in 12 of 17 (70.6\%) patients, including 9 of $12(75 \%)$ in CR group. For CR group, mean DNA quantity significantly increased after ablation $(44.8 \pm 70.7$ vs. $6.7 \pm 9.5, P=0.023)$, but there was no change in quality $(P=0.136)$. The authors concluded that EUS-guided PCA induces morphological and cytological changes in the pancreatic cystic neoplasms, but none of these predicts overall imaging-defined response to ablation.
The second prospective study from a Korean center evaluated the long-term impact of EUS guided PCA in a large group of patients with PCL studied over 10 years period. ${ }^{[9]}$ They enrolled 164 patients with PCL who underwent EUS-guided cyst ablation by using Ethanol and paclitaxel. In this study, following patients were included as follows: (i) unilocular or oligolocular cyst with 2-6 septations (ii) clinically indeterminate cyst for which EUS FNA was indicated to obtain additional information and (iii) cysts that increased in size during follow-up. All patients were asymptomatic, without abdominal pain that could be ascribed to pancreatic cysts and median largest diameter was $32 \mathrm{~mm}$ (interquartile range [IQR] 26-41). Sixteen patients $(40.2 \%)$ had unilocular cysts and 98 patients $(59.8 \%)$ had oligolocular cysts.

The cytological analysis of cyst fluid was performed in $125(76.2 \%)$ of 164 samples and results were as follows: "negative" in 58 (46.4\%), "nondiagnostic" in 52 (41.6\%), "atypical cells" in 3 (2.4\%), and "mucinous epithelium" in $12(9.6 \%)$ patients. Based on the cyst fluid analysis and imaging the presumed diagnoses were as follows: $\mathrm{MCN}(n=71,43.3 \%), \operatorname{SCN}(n=16,9.8 \%)$, IPMN $(n=11,6.7 \%)$, pseudocyst $(n=3,1.8 \%)$, and indeterminate cysts $(n=63,38.4 \%)$ patients, respectively. PCA was done using $22 \mathrm{G}$ needle and thereafter patients were followed up by a CT at 3 months and further follow-up $\mathrm{CT}$ at 6 months interval till resolution and yearly thereafter. Treatment response was assessed by comparing cyst volume at the time of PCA. Responses were arbitrarily classified as complete radiological resolution, partial resolution, or persistent cyst, corresponding to $<5 \%, 5 \%-25 \%$, and $>25 \%$ of baseline cyst volume.

As six patients were lost to follow-up, 158 patients were finally analyzed with median follow-up of 69 months (IQR 48-81). Complete resolution was observed in 114 patients (72.2\%) and partial resolution in 31 patients $(19.6 \%)$, respectively, whereas 13 patients $(8.2 \%)$ had persistent cysts. The median volume of cysts changed from $17.1 \mathrm{~mL}$ (IQR 13.1-25.3) at baseline to $3.1 \mathrm{~mL}$ (IQR 1.3-5.5) at the end of follow-up. On univariate analysis, EUS diameter $(<35 \mathrm{~mm} ; P=0.04)$ and locularity (unilocular lesion; $P<0.001)$ were significantly associated with complete resolution. The rates of complete resolution based on cystic fluid analysis were as follows: SCN, $87.5 \%$; $\mathrm{MCN}, 76.1 \%$; indeterminate cysts, $67.7 \%$; IPMN, 50.0\% $(P>0.05)$. On multivariate analysis, unilocular lesion (odds ratio [OR] 7.12, 95\% confidence interval [CI] 2.72-18.67; $P<0.001)$ and smaller cyst diameter (OR 2.39, 95\%CI 1.11-5.16, $P=0.02$ ) were 
independent predictors of complete resolution. There were 16 adverse events $(9.8 \% ; 1$ severe, 4 moderate, and 11 mild) with early adverse events being fever without documented bacteremia on blood culture $(n=1)$, pericystic spillage $(n=1)$, intracystic hemorrhage $(n=1)$, and acute pancreatitis $(n=6)$ and late adverse events (after 14 days) being pseudocyst $(n=2)$, abscess formation $(n=2)$, portal vein thrombosis $(n=1)$, splenic vein obliteration $(n=1)$, and main pancreatic duct stricture requiring endoscopic stent placement $(n=1)$.

On a follow-up of the median of 72 months, patients with initial complete resolution, radiological recurrence of the cyst was found in only two patients (1.7\%) and recurrent cysts (presumed MCN 1 and indeterminate cyst 1) were found after cyst-free intervals of 36 and 48 months, respectively. More importantly, no malignancy was discovered during follow-up. The authors concluded that among patients with pancreatic cysts in whom complete resolution was achieved after EUS-guided PCA, 98.3\% remained in remission at 6-year follow-up and unilocular form, as well as small cyst size, were predictive of complete resolution.

\section{COMMENTARY}

EUS-PCA with ethanol alone or in combination with paclitaxel has emerged as a safe and feasible alternative to surgery in the management of benign or potentially malignant cystic lesions of the pancreas. ${ }^{[5,6]}$ Previous studies evaluating the safety and efficacy of PCA have evaluated short-term response to ablation by follow-up cross-sectional imaging or surgery that has been performed after ablation. The EUS, cytological, and molecular changes following PCA have not been evaluated nor the long-term durability to the cyst ablation. The study by Kim et al. ${ }^{[8]}$ found that ablation was associated with an increase in cyst wall diameter in $68 \%$ of patients, along with decreased number or loss of septations in $39 \%$, increased internal debris in $24 \%$ and loss of mural nodule loss or calcification in $21 \%$ patients. The increase in cyst wall diameter may be due to epithelial denuding, fibrosis and chronic inflammation of the wall. They also found that ablation increased the quantity and decreased the quality of cyst fluid DNA suggesting that mutant cyst fluid DNA may be eliminated with PCA. ${ }^{[7]}$ However, none of these morphological or cytological changes predicted overall imaging-defined response to ablation. The study by Choi et al. concluded that EUS PCA has a long-term durable response rate and acceptable complications rate. ${ }^{[9]}$ The low recurrence rate of $1.7 \%$ on a long-term follow-up is reassuring and suggests that EUS-guided-PCA could be an alternative option for surgery in selected cases for the management of PCL.
Both these studies had important limitations. The study by Kim et al. used different ablative regimens for the study population that may have led to different outcomes. Furthermore, DNA changes were only looked in 17 patients treated with combined ethanol and paclitaxel and no surgical or histological samples of the treated cysts were obtained in any patient. The single arm, a noncomparative study by Choi et al. had an important limitation that the diagnosis in most of the patients was a presumptive diagnosis based on imaging and cyst fluid analysis. Moreover, as the treated patients had no or minimal risk factors for malignancy, the risk of development of malignancy associated with the ablated cysts was probably very low both at baseline and during follow-up.

Despite these limitations, both these studies are important studies that have shed light on important lacunae in our understanding of the endoscopic management of PCL, and thus EUS-PCA could be considered a possible alternative to surgery in those patients who are unfit for or refuse surgery.

\section{Ravi Sharma, Surinder Singh Rana}

Department of Gastroenterology, Postgraduate Institute of Medical Education and Research, Chandigarh, India

Address for correspondence: Dr. Surinder Singh Rana, Department of Gastroenterology, Postgraduate Institute of Medical Education and Research, Chandigarh - 160 012, India. E-mail: drsurinderrana@yahoo.co.in

\section{REFERENCES}

1. Khalid A, Brugge W. ACG practice guidelines for the diagnosis and management of neoplastic pancreatic cysts. Am J Gastroenterol 2007;102:2339-49.

2. Scheiman JM, Hwang JH, Moayyedi P. American gastroenterological association technical review on the diagnosis and management of asymptomatic neoplastic pancreatic cysts. Gastroenterology 2015;148:824-4.

3. Kiely JM, Nakeeb A, Komorowski RA, Wilson SD, Pitt HA. Cystic pancreatic neoplasms: Enucleate or resect? J Gastrointest Surg 2003;7:890-7.

4. Goh BK, Tan YM, Cheow PC, Chung YF, Chow PK, Wong WK, et al. Cystic lesions of the pancreas: An appraisal of an aggressive resectional policy adopted at a single institution during 15 years. Am J Surg 2006;192:148-54.

5. DeWitt J, McGreevy K, Schmidt CM, Brugge WR. EUS-guided ethanol versus saline solution lavage for pancreatic cysts: A randomized, double-blind study. Gastrointest Endosc 2009;70:710-23.

6. Oh HC, Seo DW, Song TJ, Moon SH, Park DH, Soo Lee S, et al. Endoscopic ultrasonography-guided ethanol lavage with paclitaxel injection treats patients with pancreatic cysts. Gastroenterology 2011;140:172-9.

7. DeWitt JM, Al-Haddad M, Sherman S, LeBlanc J, Schmidt CM, Sandrasegaran $\mathrm{K}$, et al. Alterations in cyst fluid genetics following endoscopic ultrasound-guided pancreatic cyst ablation with ethanol and paclitaxel. Endoscopy 2014;46:457-64.

8. Kim KH, McGreevy K, La Fortune K, Cramer H, DeWitt J. 
Sonographic and cyst fluid cytologic changes after EUS-guided pancreatic cyst ablation. Gastrointest Endosc 2017;85:1233-42.

9. Choi JH, Seo DW, Song TJ, Park DH, Lee SS, Lee SK, et al. Long-term outcomes after endoscopic ultrasound-guided ablation of pancreatic cysts. Endoscopy 2017;49:866-73.
This is an open access article distributed under the terms of the Creative Commons Attribution-NonCommercial-ShareAlike 3.0 License, which allows others to remix, tweak, and build upon the work non-commercially, as long as the author is credited and the new creations are licensed under the identical terms.

\begin{tabular}{|l|l|}
\hline \multicolumn{2}{c|}{ Access this article online } \\
\hline Quick Response Code: & \\
& \\
\hline
\end{tabular}

How to cite this article: Sharma R, Rana SS. Endoscopic ultrasound-guided pancreatic cyst ablation. J Dig Endosc 2017;8:206-9. 